\title{
Arbor
}

\section{Análisis filosófico de las ilusiones psicopatalógicas}

\author{
Anibal Monasterio Astobiza
}

Arbor CLXXIX, 705 (Septiembre 2004), 193-211 pp.

Durante muchos años las enfermedades mentales han sido vetadas del análisis filosófico. Afortunadamente, las modas filosóficas y el interés académico hoy ha cambiado y reconocen la importancia de la enfermedad mental (como paradigma de la disrupción mental) para el estudio del funcionamiento normal de la mente. Por esta razón, cualquier teoría contemporánea en filosofía de la mente debe tener en consideración una imagen detallada de lo que significa la parcial o total disolución de la mente. En este escrito, revisamos el aparato conceptual básico usado hasta la fecha por los filósofos para analizar y comprender las psicopatologías.

Palabras clave: filosofía psicopatológica, análisis de las ilusiones, percepción, racionalidad, intencionalidad, autoconocimiento.

"...en realidad las mayores bendiciones provienen de la locura, de hecho la locura es un regalo del cielo"

Platón, Fedro.

\section{Introducción}

La historia de la locura nos muestra la heterogénea naturaleza de una condición que ha atraído y asustado por igual. De pronto era concebida como gracia divina, inspiración de artistas, daimon filosófico que im- 


\section{Anibal Monasterio Astobiza}

pulsa a la sabiduría, como al mismo tiempo aflicción y sufrimiento individual, castigo de Dios o desequilibrio conductual pernicioso para la sociedad. La locura, la patología mental o trastorno psíquico, es fruto en muchos aspectos de una construcción social (la literatura, la pintura y contemporáneamente el cine, la prensa escrita y la TV. tienen mucho que justificar en esto) pero en nuestra opinión si que es una categoría de tipo natural y no un mito. No obstante, es evidente que durante diversos periodos históricos, la locura ha sido la excusa para el uso abusivo del poder institucional, muchas veces dominado por una epistéme o discursos elaborados y motivados por causas inconfesables que han producido la borrosa imagen de los límites de la cordura (Véase, para un análisis detallado Lanceros 1996 y Fernández Liria 2003). Enemigos del estado, rivales políticos, vagabundos, alcohólicos, idiotas, sifilíticos, hijos desheredados, prostitutas eran sospechosamente apartados acusados de dementes. Aún más, durante la cristiandad europea la lucha contra movimientos heréticos en el seno del catolicismo vio el uso indiscriminado de dictámenes de insania. Solo el incremento en el conocimiento científico de la mente/cerebro ha podido perfeccionar la atribución de signos y síntomas que a lo largo de los siglos se ha visto como estados clínico-infecciosos eran tratados como desordenes psíquicos y viceversa, severos trastornos como rasgos idiosincrásicos de personalidad. Pero no nos engañemos, la locura existe y es positivamente ${ }^{1}$ identificable. Aunque han jugado con ventaja quienes no han querido reconocerla. Las causas de este juego desigual engrosa una lista muy extensa para mencionarlas aquí, pero una no se nos ha de escapar: la elusiva naturaleza de la mente. Incluso hoy en día con el estado actual del arte en psiquiatría, neuropsicología, neuroimagen, psicofármacologia y geonómica, sigue generando batallas de expertos una definición de pongamos el caso conciencia, percepción, sensación y no digamos el tema de este escrito; la ilusión psicopatológica.

Gracias o a pesar de este panorama, el análisis filosófico ha prestado recientemente una gran atención a los trastornos mentales. Principalmente hacia aquellos que por sus características e implicaciones tienen una mayor relación con los tópicos de estudio habituales en la arena y agenda filosófica. Las ilusiones psicopatológicas son de este tipo. Estas guardan una estrecha relación con la percepción de la realidad, racionalidad (debate constreñimiento procedimental/de contenido), intencionalidad o agencia, auto-conocimiento... áreas todas ellas que trataremos en las siguientes secciones. Nuestro plan va a ser reconocer el primer tratamiento explicito contemporáneo de los trastornos mentales por parte de 
un filosofo, que reconoce la importancia del análisis filosófico de los trastornos mentales para dar cuenta de la mente en su conjunto (aunque no directamente vinculado con las ilusiones si que marca un punto de inflexión y por ello lo mencionamos). Las ilusiones y su efecto en los fenómenos básicos de la mente antes mencionados (percepción, racionalidad etc.) serán nuestro siguiente tema de revisión. Por ultimo, presentaremos a la luz de los últimos avances en neurociencia, psiquiatría evolucionista y filosofía de la mente un estatuto epistemológico de la ilusión poco intuitivo que no solo ofrece una imagen continuista entre el estado normal y el estado patológico, sino que al estado patológico lo concibe como una adaptación.

\section{El primer caso de análisis filosófico}

De acuerdo con Metzinger (2003 p. 523) Daniel Dennett y el psicólogo N. Humphrey fueron los pioneros en el análisis filosófico de las psicopatológias. Ambos autores en 1989 presentaron un análisis de una patología concreta, la cual por aquel entonces se conocía como «desorden múltiple de personalidad» que actualmente conocemos por «desorden disociativo de identidad». En este articulo seminal que llevaba por titulo Speaking for ourselves: An assesment of multiple personality disorder publicado en Raritan, Dennett despliega su teoría de la conciencia otorgando un papel esencial al centro de gravedad narrativa que se supone viene a ser el «yo», espacio de narración discursiva y apariencias estructuradas en juicios reportados lingüísticamente que dan pie a la heterofenomenologia de los demás; bajo el asesoramiento del no menos eminente psicólogo codescubridor del fenómeno de la «visón ciega» $\mathrm{N}$. Humphrey. Aquí Dennett y Humphrey describen a una paciente de nombre Mary que presentaba una infancia traumática llena de abusos físicos y represiones. Tras un periplo clínico con diversos diagnósticos fallidos, Mary tuvo suerte de toparse con un psicoterapeuta que durante una de sus sesiones observo que su diario parecía estar escrito por más de una persona. Había una personalidad para la coquetería, Sally la coqueta, para la agresión con Hatey y la sumisa Peggy. Esta plétora de personalidades que se sucedían durante las sesiones de hipnosis (tratamiento elegido por el especialista tras descubrir en su diario la división de su personalidad) guardaba un ego aún mucho más desconcertante para el análisis de Dennett y Humphrey. Sandra, la personalidad más fuerte, recordaba haber sido violada por su padrastro. En realidad, Mary constru- 
yo esta personalidad con los recuerdos más vivos y duros de su infancia traumática, con la intención no-consciente de dejar un "espacio personal» libre y ausente de conflicto emocional a través del cual dar sentido a sus experiencias. El ejemplo utilizado para el análisis filosófico casa de un modo directo con el modelo de la conciencia de Dennett etiquetado por él como «modelo de versiones múltiples», que en el caso de Mary esta afectado y no permite la integración coherente de los distintos atributos de la memoria y emociones en una experiencia consciente e integrada que no tiene porque concebirse en un sentido Cartesiano y teatral. Pero aunque pensemos en la selección del caso de Mary como el más recomendable para la teoría de Dennett no cabe duda que enfatiza un problema filosófico de base: la naturaleza del yo. Si el modelo de Dennett es una concepción que rechaza la presencia de un locus cerebral que integre unitariamente la conciencia, apoyando más bien una competición neuronal distribuida por todo el cerebro que va tejiendo una especie de telar penelopiano con ayuda de las relaciones socio-culturales; es difícil no caer en la perplejidad: entonces qué se disgrega, qué se desintegra, qué se disocia en el modelo de espacio consciente personal de Mary. Nosotros no pretendemos abordar en este escrito proyectos metafísicos de gran calado como la concepción de sentido interno o ipseidad. Aqui nos remitiremos a una área de la psicopatología, las ilusiones, mucho más modesta para el análisis filosófico que a continuación iremos presentando. Sin embargo, no hay que olvidar la importancia del sujeto, del yo, como anfitrión real o virtual de los contenidos mentales sean estos ilusiones o no, que Dennett y Humphrey en el primer caso de análisis filosófico de las psicopatologias reconocen. Para un estudio metafísico en profundidad sobre el "yo» aludimos a la obra ya mencionada de T. Metzinger. Por tanto mucho más en relación con las ilusiones y después de esta digresión histórica, vamos a centrar la atención en las ilusiones y sus efectos en fenómenos básicos de la mente tales como la percepción, racionalidad, intencionalidad o agencia y autoconocimiento ${ }^{2}$.

\section{Fenómenos mentales básicos alterados por las ilusiones}

\subsection{Percepción}

La percepción es un fenómeno relacionado con las ilusiones y es el proceso de organización de la información estimular. Nosotros únicamente nos referiremos a la percepción visual y cuando proceda a la per- 
cepción acústica, aunque hay otra serie de modalidades perceptivas que también sucumben a las ilusiones ya sean ilusiones táctiles, gustativas o corporales propioceptivas. Las ilusiones visuales son quizás los casos paradigmáticos por antonomasia de la ilusión psicopatológica. La ilusión visual se define en óptica como la percepción errónea de un estimulo externo, cuyos mecanismos psicofísicos estudiados por la psicología apuntan a un nivel estimular bajo que no activa el umbral de recepción y por tanto no se percibe correctamente, falta de atención consciente o estados de animo alterados. La ilusión en este escrito, será equiparada con la noción de alucinación en psiquiatría, es decir, como la percepción sin estimulo externo que estimula el órgano sensitivo, experimentadas como reales y no imaginarias y provenientes del exterior. Asumiendo esta definición de ilusión, tenemos que la ilusión según las teorías de la percepción clásicas en filosofía (la teoría causal de la percepción) son o bien una distorsión endógena durante el acceso intencional al contenido mental de la representación consciente del mundo, o bien una perturbación exógena intrínseca a las propiedades físicas de la escena visual ilusoriamente percibida. La formulación de la teoría causal de la percepción dice:

Un sujeto $\mathrm{S}$ percibe un objeto $\mathrm{O}$ si y solo si la percepción de $\mathrm{S}$ esta causada por la presencia de $\mathrm{O}$.

Entonces tenemos, que como distorsión endógena el sujeto no representa adecuadamente la realidad, porque su acceso consciente al contenido de sus representaciones son engañosos. Dicho de otro modo, cree estar teniendo una experiencia visual cuando en realidad no es así. En la perturbación exógena en lugar del sujeto los responsables de la ilusión son las condiciones físicas de la escena visual, los objetos y su comportamiento fotoico debido a su composición material. Un ejemplo bien conocido es el palo sumergido en agua y su reflejo torcido. La primera clase de ilusión, la endógena, es la que más nos interesa. En primer lugar, porque se equipara a la noción de alucinación en psiquiatría como hemos comentado y en segundo lugar porque a diferencia de la perturbación exógena esta no es en la mayoría de los casos corregible. Mientras que en la perturbación exógena el sujeto gracias a los principios físicos invariables que le permiten interpretar la realidad y dar coherencia a la ontología del mundo le aseguran que el palo es recto en lugar de torcido, aunque lo veamos torcido durante su introducción en el agua; las ilusiones de origen endógeno no solo son consideradas reales sino impenetrables o mejor dicho sin la capacidad de ser revisadas.

El argumento de la incorregibilidad fue por primera vez expuesto por R. Rorty (1970). Actualmente, la posibilidad de penetrar la actividad cog- 
nitiva o mejor dicho de acceder a ella de un modo intencional siendo incluso el individuo capaz de subvertir una operación cognitiva, es el centro de atención de varios filósofos (Campbell 2004; Rowlands 2004) $\mathrm{Su}$ importancia es vital para la neurociencia cognitiva y los debates sobre la arquitectura modular del cerebro (Véase, para un enfoque filosófico Fodor 1983) y también para (directamente conectado con nuestro propósito) conocer el grado de autonomía personal y subpersonal, autoconocimiento y nivel introspectivo de las personas.

Con respecto a las ilusiones de origen endógeno, estas principalmente se producen por la imprecisa representación de las experiencias privadas. Pero la cuestión es ¿qué impide, a tenor de la teoría causal de la percepción como modelo de percepción, establecer los vínculos causales entre la presencia de un objeto y su representación consciente?. ¿Es posible que el acceso intencional o cognitivo afecten a los contenidos conscientes alterando sus propiedades? Susan Hurley (1998) diferencia varios tipos de autoconciencia. La re flexividad, la primera de ellas, es la capacidad para que los contenidos conscientes se reflejen a si mismos. La segunda, la autoevidencia, permite que un sujeto acceda cognitivamente a los contenidos conscientes. De acuerdo con Hurley, la reflexividad no es suficiente para la autoconciencia. El problema como estamos viendo, es que la autoevidencia para el caso de los contenidos o propiedades de nuestras objetos reprsentacionales no es inmune a la falibilidad o lo que es lo mismo a la incorregibilidad en el sentido rortyano. Porque como bien dice Hurley (1998 p. 158) un sujeto es posible que sea consciente de $\mathrm{p}$ y se forme una creencia de que es consciente de $\mathrm{p}$, cuando en realidad no es así. Hurley continua diciendo «una creencia acerca de una experiencia visual no entraña que tu tengas esa experiencia. Contrariamente, la ausencia de una experiencia visual no entraña la ausencia de una creencia de tener una experiencia visual» ( Hurley O. c., p. 159). La anosognosia es un ejemplo de todo esto y que Hurley también menciona. La anosognosia es la negación de estar padeciendo una enfermedad o déficit. Para el caso de la visión, se conoce como el síndrome de Antón y los pacientes que no pueden ver afirman estar viendo. Pero el hecho de estar en posesión de un estado mental cuyo contenido esta falsamente representado no es el mayor de los problemas a la hora de entender la posibilidad de las ilusiones en el sentido en el que las hemos descrito. La falta de incorregibilidad que Hurley sostie ne hacia una de las formas de autoconciencia es cierta, pero para el caso de nuestra fenomenológica o lo que sentimos que es una percepción, parece ser inmune a la corrección. Metzinger (O. c., p 587) arguye que la incorregibilidad rortyana para el momento fenomenológico que estamos viviendo ahora, es cierta. Ningún neurocientífico nos pude mostrar que sea falso que el con- 
tenido de nuestros estados fenoménicos es tal y como los estamos sintiendo y que además sabemos que los estamos sintiendo. El problema que hemos ido destapando, ahora se nos duplica. La ilusión que sufre un enfermo mental (individuo delirante) es porque su contenido $u$ objeto representado es inexistente o porque su experiencia cualitativa de estar en posesión de un contenido mental, es incorrecta. O complicándolo aun más, puede ser que el objeto de la representación siguiendo las pautas de la teoría de la percepción causal, sea ilusorio; al mismo tiempo que el estado fenomenológico o el ser de estar sintiendo esa representación ilusoria sea del mismo modo ilusorio ${ }^{3}$. Por no mencionar que hay sujetos que sufren lo que se denomina intermetamorfosis inversa, la creencia de que ha habido un cambio físico o psicológico en uno mismo. Donde se puede dar el caso de no ya tener una representación consciente ilusoria o un estado fenomenológico falso sino también que yo no este sintiendo mis propias representaciones ilusorias o estados fenomenológicos falsos, sino los de otro. Y si esto no es lo suficientemente complejo, para lo que nos toca con las ilusiones visuales, recientes hallazgos en neurofisiología señalan el hecho de la equivalencia funcional entre la percepción y la imaginación. Es decir, los mismos circuitos neuronales que se activan dur rante la percepción de un objeto son los mismos que se activan cuando imaginamos el objeto. La estimulación interna durante la imaginación es la mis ma que durante la percepción de un estimulo externo (Kosslyn 1994). Entonces resulta que no hay nada que te diferencie a ti (si es que eres normal) de un paciente aquejado de ilusiones visuales. La percepción normal, la imaginación y la ilusión se superponen en lo que respecta a sus propiedades características y resulta difícil distinguirlas. (Vogeley 1999).

Las salidas a esta serie de puzzles filosóficos sobre la posibilidad de la ilusión en nuestras representaciones de la realidad pasan por el punto medio entre los informes verbales de los individuos delirantes y la investigación empírica del cerebro o lo que es lo mismo, el programa de investigación conocido como neurofenemenologia iniciado por el fallecido $\mathrm{F}$. Varela. Este programa se hace eco de un externalismo semántico, una cognición situada o encarnada que diluiría los estados internos en el ambiente desdibujando las fronteras de nuestros vehículos de representación más allá de nuestra «piel» y «cabeza».

\subsection{Racionalidad}

Si la razón es la capacidad para el pensamiento lógico y ordenado, la racionalidad es la razón entendida desde un punto de vista socio-cultural 
e individual. El caso es, que aquellos individuos que sufran estados delirantes (ilusorios) muestran una razón ilógica, o por lo menos eso dice la creencia popular. La manía persecutoria o ilusión paranoica es un ejemplo de irracionalidad. Estos individuos llegan a un punto en el cual cualquier tipo de señal, gesto o acción de una persona es interpretada como indicativo de estar siendo objeto de la intención malévola de los demás. Los marcos conceptuales básicos con los que los filósofos han entendido y entienden la racionalidad y se sirven de ellos para explicar la irracionalidad en su forma ilusoria son: el modelo de racionalidad procedimental y el modelo de la racionalidad por contenido (Gold y Hohwy 2000).

El primero de ellos, el modelo procedimental, desde Hume se entiende como aquellas reglas o pasos que aplicados sobre la información pertinente de un modo genuino nos llevan hasta cierta conclusión indefectiblemente. Este modelo llega a formar ciertos axiomas. Si dos sujetos partiendo de la misma información aplican dichas reglas, llegarán a la misma conclusión. El modelo procedimental de la racionalidad es por tanto universal. Y consiste en la aplicación procedimental y correcta de las reglas, no importa el contenido. De ahí la famosa frase de Hume: «no es contradictorio para la razón preferir la destrucción del mundo entero para arrascarme el dedo» (Gold y Hohwy 2000 p. 147). Porque para el análisis de la razón según el modelo procedimental, no es relevante el contenido de nuestros pensamientos. En cambio, es esencial para el modelo de la racionalidad por contenido, el contenido concreto de nuestros pensamientos. Para este modelo los deseos o creencias son tanto racionales o irracionales en si mismos independientemente del mecanismo instrumental que se aplique para satisfacerlos. Si yo deseo que mi gato hable, esté es un deseo y por tanto un contenido irracional. Los defensores de este último modelo entienden que por mucho aplicar reglas procedimentales la razón no es más razón, el contenido es importante. Ahora bien, qué falla en dichos modelos en la ilusión visual o acústica. Desde una explicación que parta del modelo procedimental, para el caso de una ilusión visual, el "procedimiento» no puede verse como un elemento deliberativo o reflexivo propio de las habilidades cognitivas del sujeto que no es aplicado correctamente o por lo menos si entendemos la visión en su variante simple por oposición a visón epistémica ${ }^{4}$. Para el caso de una ilusión acústica el mismo problema sobresale. Situándonos en los parámetros de la explicación del modelo de racionalidad por contenido, la ilusión visual se explicaría si todas las ilusiones visuales que un individuo sufriera tuvieran el mismo objeto visual. La ilusión se produciría porque habría una desviación desde la representación heterogénea de mundo ha- 


\section{Análisis filosófico de las ilusiones psicopatológicas}

cia la única representación de la ilusión. Pero este no es el caso. Se sabe que sujetos con ilusiones visuales creen ver tanto quimeras, dragones, bichos, extraterrestres... todo en un mismo individuo y durante el periodo más acusado de su episodio delirante. Todo esto parece indicar que ambos modelos tienen grandes limitaciones a la hora de ofrecer una explicación de la irracionalidad ilusoria, por mucho que puedan servir para explicar la racionalidad óptima. Una vía alternativa de explicación de la emergencia de la ilusión puede ser el fallo discreto de ciertos estados físicos a nivel subpersonal (estructuras y mecanismos cerebrales). Ante esto, el nivel personal (criterios normativos de determinación de los estados mentales) quizás no tengan nada que decir. Para explicarlo mejor, más arriba hemos visto como para el caso de la percepción, la autoevidencia como acceso cognitivo a los estaos conscientes, es una fuente de origen de la ilusión. Si yo me formo una creencia acerca de un estado visual inexistente, la ilusión esta presente. Pero esto es propio del nivel personal relacionado con criterios normativos, el modo en el que un individuo conceptualiza sus experiencias. Pero si pensamos que la ilusión emerge del nivel subpersonal, el individuo y su aparato conceptual no tie nen acceso a este nivel. Un ejemplo. Por mucho que yo quiera no puedo subvertir los procesos metabólicos que constantemente se llevan acabo en el interior de mi cuerpo. No puedo impedir que se den disparos neuronales en la corteza asociativa cada vez que veo una forma, aunque puela cambiar su apariencia a través de mi acceso intencional siempre y rando sea a un nivel personal; porque los disparos neuronales son prois del nivel subpersonal. Así tenemos que las ilusiones no se ajustan a explicación procedimental ni de contenido. Las ilusiones de la raciolad se explican en términos de fallos o errores sistémicos. Errores o sistémicos en el espacio de monitorización tanto procedimental le contenido. De esta forma se explica porque las ilusiones de ralad puedan afectar a los procedimientos de juicio y al contenido de ios.

llos sistémicos pueden analizarse a un nivel personal a partir ceorías atribucionales o estilos atribucionales. Las teorías atribucionales o estilos atribucionales son modos de explicación que las personas construyen para entender la realidad en función de sus historias personales y disposiciones biológicas que les llevan a dar prioridad a ciertas hipótesis o creencias frente a otras, evaluar ciertos datos a la luz de las creencias poseídas y descartar otros. Este proceso de generación de creencias y evaluación de creencias es la solución que los filósofos han dado a las ilusiones de racionalidad (Véase, Gerrans 2000). 


\subsection{Intencionalidad}

Por intencionalidad no entendemos el sentido técnico usado en filosofía de la mente para aludir a la direccionalidad referencial (dirigirse hacia algo) de los estados mentales, o como Brentano $(1874)^{5}$ díjera, la característica y marca de lo mental por oposición a lo físico. Por intencionalidad nos referimos a las propiedades de las acciones que son realizadas hacia la consecución de un propósito o en otras palabras a la capacidad de actuar. Aunque el termino intencionalidad tiene un sentido técnico y otro ordinario como por ejemplo es este ultimo, a su vez este ultimo se ha convertido en técnico y hoy los filósofos de la acción han desarrollado todo un arsenal de distinciones conceptuales entre ellas la intencionalidad, pero también la agencia en general, propiedad y fenomenología motor, que a continuación vamos a revisar desde el punto de vista de su alteración en la ilusión.

Una acción se describe como el resultado de varios procesos: intención, planificación, preparación y ejecución, y según la teoría causal de la acción, una acción se distingue de un mero suceso por sus antecedentes (Davidson 1963). Si yo deseo levantar el brazo y lo levanto se dice que es una acción porque ha sido dirigido hacia un propósito voluntarista: $m i$ intención de levantar el brazo. Pero si tu me agarras del brazo y me lo levantas no es una acción, es un hecho o un mero suceso no intencional. A su vez la teoría causal clásica ejemplificada por Davidson, recogía las características esenciales que todo concepto de intención debe tener y que se exponían en Anscombe (1957): acción intencional, intención durante la acción y expresión de la intención en el futuro. Es decir, el concepto de intención (estado mental) debe explicar la acción deliberada o intencional frente a un mero suceso, explicar cómo las razones (intenciones) dan lugar a la acción ejecutada y explicar futuras acciones. No obstante, había en la teoría causal de la acción clásica, una serie de vacíos que Searle (1983) cubrió. La teoría causal de la acción no explicaba porque hay algunas acciones de las cuales no tenemos una intención consciente, no explicaba el hecho de que hay algunas acciones donde no hay intención (derivación causal) y tampoco explicaba la diferencia fenomenológica entre acciones y otros movimientos corporales. La primera limitación se resuelve en los debates en torno a la intención, aludiendo a procesos noconscientes que actualmente juegan un papel muy relevante en actividades como el procesamiento de la información, emoción, autorregulación y acción que se pensaba requerían de intenciones conscientes y ahora sabemos que no es así (Uleman 2005). La segunda, se pone de manifiesto 


\section{Análisis filosófico de las ilusiones psicopatológicas}

en escenarios filosóficos que muestran como acciones paralelas que llevan al mismo resultado no son intencionales en sentido estricto. Por ejemplo el caso del sobrino que quiere matar a su tío para heredar la herencia (Searle O. c., p. 82). Un buen día este sobrino esta en su coche y durante su trayecto de vuelta a casa con la intención de matar a su tío atropella a un viandante que resulta ser su tío. Para Searle este escenario no es un asesinato intencional, porque aunque el atropello haya conducido al mismo resultado, matar a su tío, el atropello no tenía la intención de causar esa acción que trajo el resultado deseado. La última limitación de la teoría causal de la acción, que Searle refina, es la distinción entre acciones y otro tipo de movimientos a nivel fenomenológico. Searle salva este obstáculo apelando a la intención previa y la intención durante la acción. El primer tipo de intención puede o no causar una acción. Mientras que toda acción necesita una intención (consciente o noconsciente) no toda intención tiene porque ir seguida de una acción.

No obstante el modelo mejorado de Searle no cubre acciones no-intencionales donde pueden entran las ilusiones de la agencia (Perner 2003). Perner (2003 O. c. 220 y ss) ofrece para dar cuenta de este tipo de acciones su modelo de rutas duales o paralelas en el control de la acción no-intencional, como por ejemplo en el caso de las acciones automáticas, fallos normales del control... Pero nosotros repararemos en aquellas acciones que son no-intencionales y patológicas (ilusiones de la agencia) y que el modelo de Perner no explica tampoco. Como corolario final, actualmente en las discusiones filosóficas para que una acción sea intencional, dicha intención debe incluir cinco componentes: que el agente tenga un deseo por algo, una creencia de que una determinada acción le levará a conseguir ese algo, la habilidad para realizar la acción y la conciencia de que la intención conseguirá ese algo ( Malle y Knobe 1997; Malle 2004 p. 45).

Para los casos de disfunción en la intencionalidad o agencia (ilusión intencional), la esquizofrenia es la condición patológica que mayor atractivo tiene para los filósofos de la psiquiatría o filósofos psicopatológicos ${ }^{6}$. Para entender la intencionalidad y su ilusión, la esquizofrenia ${ }^{7}$ es el mejor paradigma. Obviando los aspectos neurobiológicos, y asumiendo que el lector tiene el conocimiento nosológico y de los criterios de diagnostico de la enfermedad 8 ; con relación a la conducta los filósofos han echado mano del marco elaborado por el neurocientífico Chris Frith (1992) para entender por qué los esquizofrénicos muestran una conducta no-intencional. El modelo de Frith se basa en diferenciar entre acciones que son motivados por la voluntad del individuo y las acciones que son causadas 
por elementos físicos del entorno. Usando un ejemplo suyo una acción motivada por la voluntad es el intento de una persona de aprender francés. Como esta persona desea aprender francés hará aquello que le conduzca al objetivo. En cambio, si yo estoy en un restaurante y leo la carta y dado que en la carta hay Mouse de salmón ahumado y decido tomar Mouse de salmón ahumado, esta acción esta causada por elementos del entorno (carta). Habiendo presentado estas dos clases de acciones, Frith propone un sistema de monitorización cognitiva que nos permite mantener y evaluar la información de cualesquiera de estas acciones. El caso es que los esquizofrénicos tienen funcionalmente «roto» este sistema de monitorización cognitiva, lo que les impide diferenciar entre la información de una y otra respectivamente. Así, cuando un esquizofrénico decide voluntariamente realizar una acción, pierde la información en ruta canalizada para la acción voluntaria y durante la realización de la acción hay un "impasse» que le hace pensar que la acción no estaba motivada por su propia voluntad sino por elementos del entorno. De nuevo usando uno de los ejemplos de Frith, si tenemos a un esquizofrénico hablando con un interlocutor mientras toman el café, el esquizofrénico mirará a su interlocutor para preguntarle algo pero inmediatamente «olvidará» por qué le estaba mirando especulando que quizás le esta mirando porque su interlocutor le había llamado por su nombre.

Pero las ilusiones de la intencionalidad o la agencia no se limitan a los delirios de referencia interna o externa a nivel de control de la acción, enfatizados por el modelo de Frith; también hay ilusiones de conciencia o de propiedad de la acción que son interesantes por su significado filosófico. Para profundizar en las ilusiones de conciencia o de propiedad de la acción, es decir, tener noticia consciente del movimiento iniciado por uno mismo, hay que diferenciar entre propiedad y percatación. La «percatación» es la capacidad que nos posibilita saber si la acción que realizo esta volitivamente iniciada por mí. Se sabe que hay ciertas condiciones patológicas o síndromes como por ejemplo el conocido como síndrome de dependencia ambiental (Lhermitte 1986) que imposibilita al individuo distinguir conscientemente si una acción es iniciada por él o es parte del entorno. Es como si el «yo» del sujeto se diluyera entre los diversos movimientos que podemos estar observando en una escena visual como por ejemplo el movimiento de las ramas de un árbol, el movimiento de los coches, el movimiento del resto de personas... La «propiedad» es la capacidad que nos permite decirnos a nosotros mismos si uno es el autor de la acción por medio de la conciencia de la misma. El «síndrome de la mano anárquica» es un ejemplo de déficit de propiedad (conciencia). 


\section{Análisis filosófico de las ilusiones psicopatológicas}

Individuos que padecen este síndrome se atan la camisa con una mano mientras con la otra la están desabrochando (Marcel 2003). La intencionalidad de las acciones en esta serie de casos ilusorios de acuerdo con el sentido de ilusión usado en este escrito, como vemos está cuando menos afectada.

Una de las vías tentativas de solución o por lo menos de conocimiento más profundo de la problemática de la intencionalidad y sus diversos tipos de ilusiones en filosofía, estriba en estar atento a los grandes avances en neurociencia motor. Enfoques basados en la ingeniería y teoría de control han servido para explicar los trastornos de la intencionalidad. Entre los más significativos se encuentran el modelo interno de predicción motor de Wolpert (1997).

\subsection{Autoconocimiento}

Aunque en un principio hayamos dicho que por autoconomciento no nos referiremos a la empresa metafísica de la búsqueda del yo, si que hay que tomar ciertos hallazgos de este programa de investigación para dar con lo que entendemos por autoconocimiento: disponibilidad del contenido mental, sus criterios determinantes y normativos, satisfacción y fiabilidad.

En primer lugar, del programa de investigación metafísico acerca de la naturaleza del yo, obtenemos que hay que dejar bien claro que toda apelación a un contenido cualitativo de nuestras experiencias o qualia como los filósofos gustan en decir es un sinsentido. No solo porque no hay una definición operativa valida de qualia que todo el mundo utilice, sino porque su mera observación o disponibilidad de contenido es imposible porque son inexistentes (Metzinger O.c. p. 64 y SS). Por introspección Metzinger nos habla de cuatro sentidos distintos, todos ellos con mayor o menor presencia en la tradición filosófica y literatura actual. Uno de ellos, la introspección como referencia cognitiva conscientemente experienciada, sino se hace un cuidadoso análisis se puede confundir con la auto-referencia cognitiva conscientemente experienciada. Por otra parte atendiendo a la experiencia fenoménica o qualia, nos recuerda Metzinger (O.c., p. 69) que la literatura filosófica ha pasado por alto, un hecho empírico, que el ser humano no dispone de un criterio de identidad introspectiva en términos de la segunda noción de introspección $n^{9}$ que refiera cognitivamente a los elementos simples de nuestro modelo interno del mundo. En otras palabras Metzinger niega el yo y su contenido fenomé- 


\section{Anibal Monasterio Astobiza}

nico, pero existe una cierta incongruencia. Esta presunta incapacidad en la autoascripcion para determinar el contenido cualitativo de nuestras percepciones corre paralelo o parece similar a una de las variedades de autoconciencia que más arriba hemos mencionado: la autoevidencia. Pero el caso es que para Hurley no tiene porque ser la autoevidencia incorregible y algunas de sus consecuencias son las distintas ilusiones sobre el contenido visual que hemos comentado. En cambio, si entendemos que la autoevidencia de Hurley es equivalente a la autoascripción de propiedades psicológicas de Metzinger que él mismo considera incorregibles como también hemos visto; entonces cómo es posible que sea la autoascripcion incorregible si no hay qualia ni yo que tenga experiencias. Tenemos que independientemente del método de disponibilidad o accesibilidad (introspección) ambos autores hacen referencia a dos niveles de autoconocimiento interdependientes pero autónomos. Para conceptualizarlos mejor nosotros llamaremos al primer nivel etiquetado por Hurley como auto evidencia (acceso cognitivo del sujeto al contenido de los estados concientes) inmersión representativa y al segundo nivel equivalente a la autoascripción de propiedades psicológicas del modelo de yo fenomenológico de Metzinger, inmersión fenomenológica.

En la inmersión representativa o en términos hurleyanos autoevidencia es posible la alteración del contenido de nuestros estados porque hay mediación cognitiva que distorsiona o permite un acceso transparente al contenido. En la inmersión fenomenológica o en términos de Metzinger autoascripción de propiedades uno esta en un estado o momento insustituible independiente de que haya acceso cognitivo o no. Aunque no sabemos cómo sale Metzinger de la incongruencia que hemos detectado si apelando a su modelo de fenomenológico del yo, no cabe duda que aun negando el yo o los qualia todo autoconocimiento tiene dos niveles conectados lo que nosotros llamamos inmersión representativa e inmersión fenomenológica. La ilusión en el autoconocimiento no escapa a ninguno de estos niveles. Hurley nos ha mostrado como a nivel de inmersión representativa es posible la ilusión. Pero en contra de lo que parece sugerir Metzinger, a nivel de inmersión fenomenológica también es posible la ilusión. De lo que ya no estamos seguros, dado el poder cautivador de los argumentos de Metzinger en algunos aspectos, es de la existencia de los qualia o del yo; lo que hace no solo más difícil de comprender por qué Metzinger en otros aspectos asegura la incorregibilidad de lo que nosotros llamamos inmersión fenomenológica, sino también la posibilidad misma de que haya ilusiones en el sentido usado en este escrito desviadas de una percepción, racionalidad, intencionalidad y autoconoci- 
miento normal. Porque si no existe el yo ni los qualia, entonces quién delira. Ante las perturbaciones e ilusiones en el autoconocimiento, los filósofos aun siguen dirimiendo los procesos que se ven afectados, qué enfoque tomar (primera persona o tercera persona) e incluso si en realidad hay dicho autoconocimiento en tanto y cuanto algunos piensan que el yo no es ninguna categoría de tipo natural.

\section{Discusiones}

No hace falta abrazar una doctrina metafísica que niegue la existencia del yo (Metzinger O. c.; Berrios y Marková 2003) para endosar ciertas dudas sobre la propia existencia de la ilusión o por lo menos su sentido epistemológico negativo, a pesar de las alteraciones que como hemos visto las ilusiones producen en la mente. Es decir, que no tenemos que defender una posición humeana con respecto al yo, para en su derivación lógica atenuar que la ilusión sea una disrupción de las capacidades cognitivas del sujeto ${ }^{10}$.

Sin meternos en aguas enfangadas a cerca de la existencia del yo (porque aún pensando que los argumentos de los defensores de esta postura sean cautivadores, estos tienen que mostrarnos entonces por qué tenemos la intuición de sentido común de un sujeto para pensamientos, emociones, deseos...) se puede defender la idea de la ilusión como una adaptación en lugar de un estado patológico. El modelo paradigmático que nos ha estado moviendo en la introducción a la mayoría de las ilusiones que pueden darse en fenómenos mentales básicos como la percepción, racionalidad, intencionalidad y el autoconocimiento, a la par de las diversas concepetualizaciones filosóficas que para entenderlas se han elaborado (que no es otro que el trastorno mental de la esquizofrenia) nos sirve de igual modo como ejemplo para comprender la naturaleza adaptativa de las ilusiones y patologías en general. En primer lugar, la patología (ilusión para el propósito de este escrito) es una función adaptativa biológica. No es una condición antitética a un estado de bienestar físico/mental y social, es una continuación, es la respuesta orgánica ante situaciones percibidas como peligrosas para el sujeto. Brendan Maher las define como creencias falsas que emergen como respuestas racionales ante experiencias inusuales. Volviendo al modelo de la esquizofrenia como fuente de las más desconcertantes ilusiones, el enfoque de la patología como adaptación cobra toda su virtualidad y credibilidad. Porque la esquizofrenia, el más severo de los trastornos psiquiátricos con un ratio de pre- 
valencía poblacional de 1 a 100 que afecta por igual a hombres y mujeres que se manifiesta en la ultima etapa de la adolescencia o el inicio de la edad adulta y que no se encuentran diferencias epidemiológicas en su incidencia cultural afectando a culturas preliterarias, literarias, agrícolas e industriales; nos conduce a un problema que se denomina la paradoja darwiniana en honor a C. Darwin. Si un rasgo reduce las posibilidades de éxito reproductivo y supervivencia de un individuo, es difícil ver como este mismo rasgo puede mantenerse en el acervo genético, esta es la paradoja darwiniana. La esquizofrenia sin lugar a dudas reduce las posibilidades de supervivencia. La pregunta ahora es por qué sigue habiendo esquizofrenia (para el propósito de este escrito ilusiones). A un nivel social las hipótesis para la permanencia son la posibilidad de un pensamiento mucho más analógico, transversal y creativo. Los individuos que sufren de ilusiones tienen una mayor habilidad para conectar semánticamente eventos a primera vista no interrelacionados. Esto a su vez les reporta un cierto carisma que se traduce en prestigio social sobre todo en aquellos ámbitos de la vida donde se requiere este tipo de pensamiento (ciencia, arte, política) lo que nos puede dar una idea de su persistencia. A nivel inmunológico se sabe que los individuos que sufren esquizofrenia tienen una mayor inmunocompetencia, es decir la capacidad para hacer frente a bacterias y parásitos. Pero lo más sorprendente es la relación entre la esquizofrenia, lateralidad hemisférica y lenguaje, relación avanzada por la hipótesis del psiquiatra oxoniense T. Crow. De entre la complejidad de sus argumentos se puede extraer que el lenguaje humano ha madurado y cristalizado gracias a una mutación genética que ha permitido la lateralización de esta función en el cerebro, pero que el precio por esta grandiosa habilidad que es el lenguaje es la esquizofrenia. En otras palabras, que debido a que existe la esquizofrenia tenemos lenguaje.

Pero el giro epistemológico, un giro de $180^{\circ}$, que pasa por ver la ilusión como una adaptación y no como un desorden no acaba aquí. La psiquiatría evolucionista además de mostrarnos la naturaleza adaptativa de las ilusiones nos dice que es posible encontrar trastornos mentales en otros animales, lo que supone para la filosofía de la mente consolidar aún más la idea de no concebir cada una de las facultades mentales humanas como exclusivas y únicas del ser humano, incluso para el caso de su funcionamiento anormal. Y mucho más, para una mejora en el tratamiento clínico pero quizás más importante, para desterrar la imagen negativa y estigmatizante del enfermo mental o individuo delirante; debemos pensar como ya hemos dicho anteriormente que no hay diferencia significativa entre la percepción normal, la imaginación y la ilusión. Tal y como 


\section{Análisis filosófico de las ilusiones psicopatológicas}

dice Metzinger (O. c., p. 51) nuestra experiencia fenoménica cuando estamos conscientes y despiertos no es más que una alucinación en-línea. Y sobre lo dicho, una revisión no constructiva sino descriptiva dado la escasa tradición en filosofía psicopatológica en España (exceptuando López-Ibor 1993), cerramos el círculo en el mismo punto donde empezamos:

«En realidad las mayores bendiciones provienen de la locura, de hecho la locura es un regalo del cielo» Platón, Fedro

\section{Notas}

1 Positivo es usado en su sentido etimológico de referencia a lo «dado» o «hecho físico». En este caso, el correlato anatómico neuronal.

2 Autoconocimiento no como búsqueda de un «yo» que como hemos dicho lo dejamos para empresas metafísicas mas relevantes que Metzinger y otros (Gallagher 2000) actualmente pergeñan. Autoconocimiento, como un modo de saber de la disponibilidad del contenido mental, sus criterios determinantes y normativos, satisfacción y fiabilidad.

3 Una distinción entre inmersión representativa e inmersión fenomenológica que más adelante en el escrito definiremos.

4 Bermúdez (2003 p. 174) define la visón epistémica como aquella visión que crea contextos opacos y que se establece sobre la base de relaciones epistémicas entre el perceptor y las proposiciones. Por el contrario la visón simple la define como aquella visión que crea contextos trasparentes y se establece por relación directa entre los perceptores y los objetos.

5 Es en su obra Psicología Desde un Punto de Vista Empírico donde reevalúa la concepción medieval de «esse intentionale» (objetos de la mente), Brentano formula la noción moderna de intencionalidad que mas tarde hará suya la escuela fenomenológica.

${ }^{6}$ Aunque no hay que olvidar que los síndromes del espectro Autístico entre los que se encuentra el propio autismo, síndrome de Asperger, síndrome de Rett y Déficit de Atención Hiperactivo, también son estudiados filosóficamente por las dificultades que muestran las personas que lo sufren en relación a la intencionalidad, teoría de la mente y en definitiva el buen funcionamiento social.

7 No deben olvidar los lectores que en un sentido laxo y no ampliamente delimitado pero identificable cada vez que hablemos de esquizofrenia a lo largo del texto, se hace mención a uno de sus síntomas: las ilusiones. Las cuales son interpretadas en su sentido alucinatorio y no óptico y como estas a su vez afectan la mente objeto de estudio de la filosofía. Es decir esquizofrenia e ilusión se usara intercambiablemente.

8 Si no es el caso, para entender las bases neurobiológicas de la esquizofrenia véase, Albert et al. 2002. Para un buen conocimiento nosológico y de los criterios validos de diagnostico Fauman 2003.

9 Haciendo honor al legado kantiano Metzinger realiza un análisis trascendental de nuestra mente y diferencia como hemos comentado, cuatro nociones de introspección: 1) atención externa 2) referencia cognitiva conscientemente experienciada 3) percepción interna 4) auto-referencia conscientemente experienciada.

10 Porque si pensáramos que no hay un «yo» como bien hemos expuesto en la sección anterior, entonces cómo se puede estar delirando o mejor dicho quién delira. 


\section{Bibliografia}

ALBERT K. A. et al. (2002): Evidence for decreased DARPP-32 in the prefrontal cortex of patients with schizophrenia. Arch. Gen. Psychiat., 59, 705-712.

ANsCOMBE G. E. (1957): Intention. Oxford: Blackwell.

BERMUdEZ J. (2003); Thinking without words. Oxford: Oxford University Press.

Berrios G. y MARKovÁ I. (2003): The self and psychiatry: A conceptual history. En Tilo Kircher y Anthony David (eds) The Self in Neuroscience and Psychiatry. Cambridge: Cambridge University Press.

CAMPBELl J. (2004): Molyneux's question and cognitive impenetrability. En Athanassios Raftopoulos (ed) Cognitive Penetrability of Perception: Attention, Action, Strategies and Bottom-Up Constraints. New York: Nova.

DAvidson D. (1963): Actions, reasons and causes. Journal of Philosophy, 60, 685-700.

FAUMAN M. (2003): DSMV-IV-TR: Guía de Estudio. Barcelona: Masson.

FERNÁNDEZ LIRIA A. (2003): Conceptos sustantivo y pragmático de la enfermedad mental. Implicaciones clínicas. En Enrique Baca y José Lázaro (eds) Hechos y Valores en Psiquiatría. Madrid: Triacastela.

FoDOR J. (1983): The Modularity of Mind. Massachusetts: MIT Press.

FRITH C. (1992): The Cognitive Neuropscyhology of Schizophrenia. Hove: Lawrence Erlbaum.

GALlaGHER S. (2000): Philosophical conceptions of the self: Implications for cognitive science. Trends in Cognitive Science, 4, 14-21.

GerRans P. (2000): Refining the explanation of Cotard's Delusion. En Max Coltheart y Martin Davies (ed) Pathologies of Belief. Oxford: Blackwell publishers.

GoLD I. y HoHwy J. (2000): Rationality and schizophrenic delusion. En Max Coltheart y Martin Davies (ed) Pathologies of Belief. Oxford: Blackwell publishers.

HURLEY S.L. (1998): Consciousness in Action. Massachusetts: Harvard University Press.

Kosslyn S.M. (1994): Image and Brain: The Resolution of the Imagery Debate. Massachusetts: MIT Press.

LANCERos P. (1996): Avatares del Hombre: El Pensamiento de Michel Foucault. Bilbao: Universidad de Deusto.

LhermitTe F. (1986):Human anatomy and the frontal lobes. Part II: Patient behaviour in complex and social situations : The «environmental dependency syndrome». Annals of Neurology, 19, 335-343.

LOPEZ-IBOR J. (1993): La personalidad en medicina y sus trastornos. Discurso de Ingreso o Discurso de Toma de Posesión: Real Academia Nacional de Medicina, España.

MALle B. y KnOBE J. (1997), The Folk Concept of intentionality. Journal of Experimental Social Psychology, 33, 101-121.

MALlE B. (2004): How the Mind Explains Behaviour: Folk Explanations, Meaning and Social Interaction. Massachusetts: MIT Press.

MARCEL A. (2003): The sense of agency: Awareness and ownership of action. En Johannes Roessler y Naomi Eilan (eds) Agency and Self-Awareness. Oxford: Oxford University Press.

Metzinger T. (2003): Being No One: The Self-Model Theory of Subjectivity. Massachusetts: MIT Press.

PERNER J. (2003): Dual control and the causal theory of action: The case of non-intentional action. En Johannes Roessler y Naomi Eilan (eds) Agency and Self-Awareness. Oxford: Oxford University Press. 


\section{Análisis filosófico de las ilusiones psicopatológicas}

RORTY R. (1970): Incorrigibility as the mark of the mental. Journal of Philosophy 67, 406424.

Rowlands M. (2004): The cognitive penetrability of perception. En Athanassios Raftopoulos (ed) Cognitive Penetrability of Perception: Attention, Action, Strategies and Bottom-Up Constraints. New York: Nova.

SEARLE J. (1983): Intentionality. Cambridge: Cambridge University Press.

ULEMAN J. (2005): Introduction: Becoming aware of the new unconscious. En Ran R. Has sin, James S. Uleman y John A. Bargh (eds) The New Unconscious. Oxford: Oxford University Press.

VOGELEY K. (1999): Hallucinations emerge from an imbalance of self monitoring and reality modelling. The monist, $82,626-44$.

WOLPERT D. (1997): Computational approaches to motor control. Trends in Cognitive Sciences, 1, 209-216. 